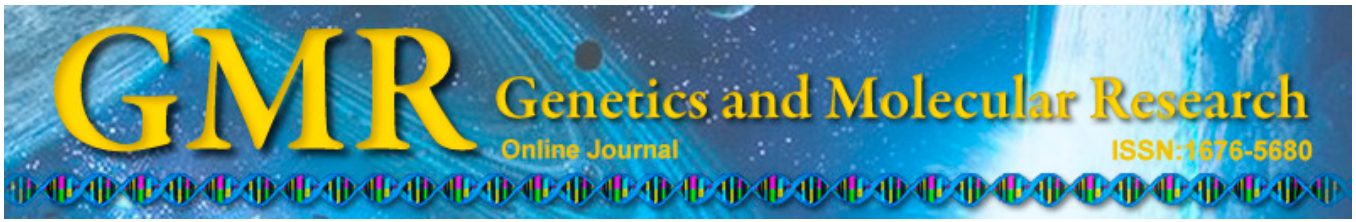

\title{
Recombinant adenovirus-mediated overexpression of PTEN and KRT10 improves cisplatin resistance of ovarian cancer in vitro and in vivo
}

\author{
H. Wu, K. Wang, W. Liu and Q. Hao \\ Key Laboratory of Cancer Prevention and Therapy, \\ Department of Gynecological Oncology, \\ Tianjin Medial University Cancer Institute and Hospital, Tianjin, China \\ Corresponding author: Q. Hao \\ E-mail: quan_haotj@126.com
}

Genet. Mol. Res. 14 (2): 6591-6597 (2015)

Received January 10, 2014

Accepted May 26, 2015

Published June 18, 2015

DOI http://dx.doi.org/10.4238/2015.June.18.1

\begin{abstract}
Drug resistance is a major cause of treatment failure in ovarian cancer patients, and novel therapeutic strategies are urgently needed. Overexpression of phosphatase and tensin homolog (PTEN) has been shown to preserve the cisplatin-resistance of ovarian cancer cells, while cisplatin-induced keratin 10 (KRT10) overexpression mediates the resistance-reversing effect of PTEN. However, whether overexpression of PTEN or KRT10 can improve the cisplatin resistance of ovarian cancer in vivo has not been investigated. Therefore, we investigated the effects of adenovirus-mediated PTEN or KRT10 overexpression on the cisplatin resistance of ovarian cancer in vivo. Recombinant adenoviruses carrying the gene for PTEN or KRT10 were constructed. The effects of overexpression of PTEN and KRT10 on cisplatin resistance of ovarian cancer cells were examined using the 3(4,5-dimethylthiazol-2-yl)2,5diphenyltetrazolium bromide (MTT) and TdT-mediated dUTP nickend labeling (TUNEL) assays in vitro. Subcutaneously transplanted
\end{abstract}


nude mice, as a model of human ovarian cancer, were used to test the effects of PTEN and KRT10 on cisplatin resistance of ovarian cancer in vivo. The MTT assay showed that recombinant adenovirus-mediated overexpression of KRT10 and PTEN enhanced the proliferation inhibition effect of cisplatin on $\mathrm{C} 13 \mathrm{~K}$ cells. Recombinant adenovirusmediated overexpression of KRT10 and PTEN also increased the cisplatin-induced apoptosis rate of C13K cells. Furthermore, recombinant adenovirus-mediated overexpression of KRT10 and PTEN enhanced the inhibitory effect of cisplatin on C13K xenograft tumor growth. Thus, recombinant adenovirus-mediated overexpression of KRT10 and PTEN may improve the cisplatin resistance of ovarian cancer in vitro and in vivo.

Key words: Cisplatin; Cisplatin-induced keratin 10; Ovarian cancer; Multi-Drug Resistance; Phosphatase and tensin homolog

\section{INTRODUCTION}

Ovarian cancer, one of the most common gynecologic malignancies, has the highest mortality rate among all gynecologic malignancies. Currently, surgery and platinum-based chemotherapy are the primary methods for treating ovarian cancer. Although $75-80 \%$ of ovarian cancer patients respond to chemotherapy initially, more than $80 \%$ of patients who undergo chemotherapy display drug resistance, and even multi-drug resistance (MDR), ultimately leading to poor prognosis. This suggests that MDR is a common cause of chemotherapy failure in ovarian cancer (Stordal et al., 2007). Therefore, developing targeted treatment methods for reversing MDR is very important for treating ovarian cancer.

Phosphatase and tensin homolog (PTEN), a tumor suppressor gene, plays a crucial role in cell apoptosis, cell cycle arrest, and cell migration (Li et al., 1997; Dahia, 2000). Chemotherapy drugs, such as cisplatin, can induce the apoptosis of ovarian cancer cells by enhancing PTEN activity (Yan et al., 2006; Wan et al., 2007; Li et al., 2011; Singh et al., 2013). These studies indicate that overexpression of PTEN improves the MDR of ovarian cancer (Steelman et al., 2008; Wu et al., 2008; Singh et al., 2013). In our previous study, we showed that overexpression of PTEN reversed cisplatin resistance in the ovarian cancer cell line $\mathrm{C} 13 \mathrm{~K}(\mathrm{Wu}$ et al., 2008). Further examination demonstrated that cisplatin-induced keratin 10 (KRT10) overexpression in $\mathrm{C} 13 \mathrm{~K} / \mathrm{PTEN}$ ovarian cancer cells mediated the resistance-reversing effect of PTEN. However, whether overexpression of PTEN or KRT10 also improves the cisplatin resistance of ovarian cancer in vivo has not been investigated. Therefore, in the present study, we first constructed recombinant adenoviruses carrying PTEN or KRT10 genes and then investigated their effects on the cisplatin resistance of ovarian cancer in vivo.

\section{MATERIAL AND METHODS}

\section{Cell culture}

The cisplatin-resistant ovarian cancer cell line C13K was cultured in RPMI-1640 supplemented with $10 \%$ fetal bovine serum (FBS), $100 \mathrm{U} / \mathrm{mL}$ penicillin, and $100 \mathrm{U} / \mathrm{mL}$ strep- 
tomycin at $37^{\circ} \mathrm{C}, 5 \% \mathrm{CO}_{2}$, and $95 \%$ humidity. The three-dimensional (3D) ovarian cancer culture model was prepared according to a previous study (Smith et al., 2011).

\section{Construction of recombinant adenoviruses}

PTEN and KRT10 genes were inserted into the pacAd5-CMV-IRES-GFP vector to prepare shuttle vectors. HEK293 cells $\left(2 \times 10^{6}\right.$ cells/well) were seeded in 6-cm dishes in Dulbecco's modified Eagle medium (DMEM) supplemented with 10\% FBS and incubated at $37^{\circ} \mathrm{C}$ with $5 \% \mathrm{CO}_{2}$ overnight. The medium was replaced before transfection. The cells were transfected at 80-90\% confluence with pacAd5-9.2-100 and shuttle plasmids pacAd5-CMVIRES-GFP/KRT10 or pacAd5-CMV-IRES-GFP/PTEN using Lipofectamine 2000 (Invitrogen; Carlsbad, CA, USA) according to manufacturer instructions. Twelve days post-transfection, recombinant adenoviruses carrying PTEN (AD-PTEN) and KRT10 (AD-KRT10) genes were harvested. All recombinant adenoviruses were amplified in HEK293 cells and purified using double cesium chloride density gradient ultracentrifugation. Titers of the adenoviral stocks were determined using a plaque assay on 293 cells.

\section{3(4,5-Dimethylthiazol-2-yl)2,5-diphenyltetrazolium bromide (MTT) assay}

Ovarian cancer cells were seeded into 96-well plates at a density of $1 \times 10^{4}$ cells/well in RPMI-1640 supplemented with 10\% FBS. Cells were treated with or without the indicated concentrations of cisplatin as: $0,2.5,5,7.5,10,12.5$ and $15 \mu \mathrm{M}$. After $24 \mathrm{~h}, 10 \mu \mathrm{L} 5 \mathrm{mg} /$ $\mathrm{mL}$ MTT solution was added to each well. The plates were incubated at $37^{\circ} \mathrm{C}$ for $4 \mathrm{~h}$; the supernatant was discarded and $100 \mu \mathrm{L}$ dimethyl sulfoxide was added to each well and mixed thoroughly before reading on a microplate reader.

\section{TdT-mediated dUTP nick-end labeling (TUNEL) assay}

After cisplatin treatment for $24 \mathrm{~h}$, the cells were fixed with $4 \%$ paraformaldehyde and embedded with paraffin using standard methods. The TUNEL assay was performed according to fluorescein in situ cell death detection kit manufacture instructions (Roche, Basel, Switzerland). Nuclei were stained using 4'6-diamidino-2-phenylindole. Four different fields from each section were analyzed. The number of TUNEL-positive cells was divided by the total number of ovarian cancer cells to determine the apoptosis rate.

\section{Establishment of a subcutaneously transplanted nude mice model of human ovarian cancer}

C13K cells $\left(4 \times 10^{6}\right.$ cells/animal $)$ were injected subcutaneously into nude mice to produce implanted tumors. Tumor volumes were measured using a slide caliper every 3 days according to the formula: volume $=($ larger diameter $) \times(\text { smaller diameter })^{2} / 2$ (Liu et al., 2008). Two weeks later, AD-KRT10, AD-PTEN, and negative adenovirus were injected into the tumors. Twenty-four hours later, $5 \mathrm{mg} / \mathrm{kg}$ cisplatin was injected through the tail vein. Tumor volumes were measured using a slide caliper every 3 days until the 16th day after cisplatin administration. 


\section{Statistical analysis}

All results are reported as means \pm standard deviation (SD). Statistical significance was determined using SPSS 17.0 for Windows (SPSS, Inc., Chicago, IL, USA). One-way analysis of variance was performed for multiple comparisons followed by Fisher's least-square deconvolution post-hoc comparisons. Differences were considered significant when $\mathrm{P}<0.05$.

\section{RESULTS}

\section{Recombinant adenoviruses carrying PTEN or KRT10 genes enhanced the chemosensitivity of $\mathrm{C} 13 \mathrm{~K}$ cells to cisplatin}

To investigate whether adenovirus expressing PTEN or KRT10 could improve the chemosensitivity of ovarian cancer cells to cisplatin, C13K cells were infected with AD-PTEN or AD-KRT10 and then seeded in a 3-D culture environment on a 96-well plate. After treatment with cisplatin for $48 \mathrm{~h}$, the MTT assay was performed. As shown in Figure 1A, both AD-PTEN and AD-KRT10 significantly enhanced the cytotoxicity of cisplatin toward C13K cells compared to control adenovirus-infected cells. AD-PTEN-infected C13K cells showed higher cisplatin sensitivity than AD-KRT10-infected C13K cells. Furthermore, the TUNEL assay showed that the apoptosis rates of AD-PTEN- and AD-KRT10-infected C13K cells were higher than that of control adenovirus-infected cells after cisplatin treatment (Figure 1B). A significant difference was also noted between AD-PTEN- and AD-KRT10-infected C13K cells (Figure 1C).

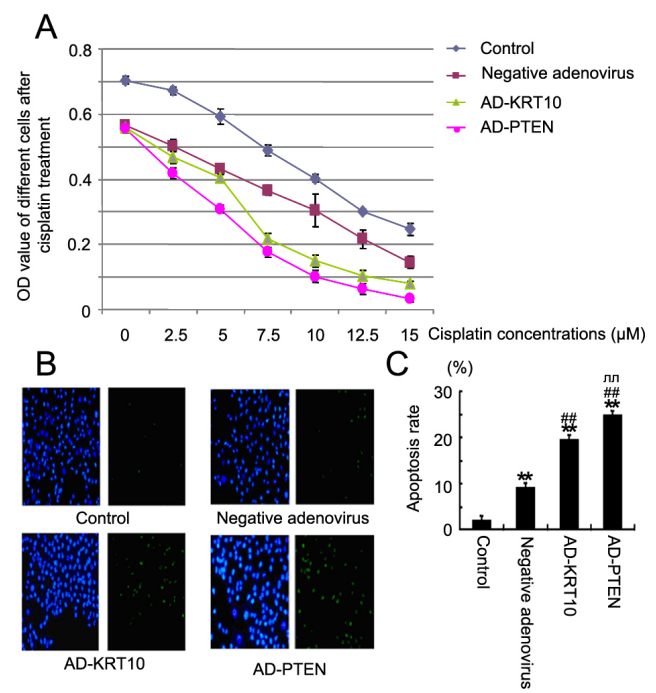

Figure 1. Recombinant adenovirus-mediated KRT10 and PTEN overexpression enhanced the sensitivity of C13K cells to cisplatin. A. KRT10 and PTEN overexpression enhanced the proliferation inhibitory effect of cisplatin on ovarian cancer cells. B. KRT10 and PTEN overexpression enhanced cisplatin-induced apoptosis of C13K cells determined by TUNEL staining. C. Apoptosis rate of C13K cells induced by cisplatin. C13K cells were infected with AD-KRT10, AD-PTEN, or negative adenovirus, and these cells were seeded into 96- and 6-well plates in a 3-D microenvironment. The MTT or TUNEL assay was performed after cisplatin treatment for 48 or $24 \mathrm{~h}$, respectively. Data are reported as means $\pm \mathrm{SD}(\mathrm{N}=3)$. ${ }^{* *} \mathrm{P}<0.01$, compared with control group. ${ }^{\#} \mathrm{P}<0.01$, compared with negative adenovirus group. ${ }^{\text {лл}} \mathrm{P}<0.01$, compared with AD-KRT10 group. 


\section{Recombinant adenoviruses carrying PTEN or KRT10 genes improved the anti-ovarian cancer effect of cisplatin in vivo}

To confirm the results obtained in vitro, we tested the in vivo effects of AD-PTEN and AD-KRT10. Tumor growth was slower in the AD-PTEN and AD-KRT10 groups than in the control adenovirus group. As shown in Figure 2A and B, 6 weeks post-cisplatin treatment, tumor volumes were much lower in the AD-PTEN and AD-KRT10 groups than in the control adenovirus group. Similarly to the in vitro results, AD-PTEN showed a more powerful effect in strengthening the anti-ovarian cancer effect of cisplatin compared to AD-KRT10.

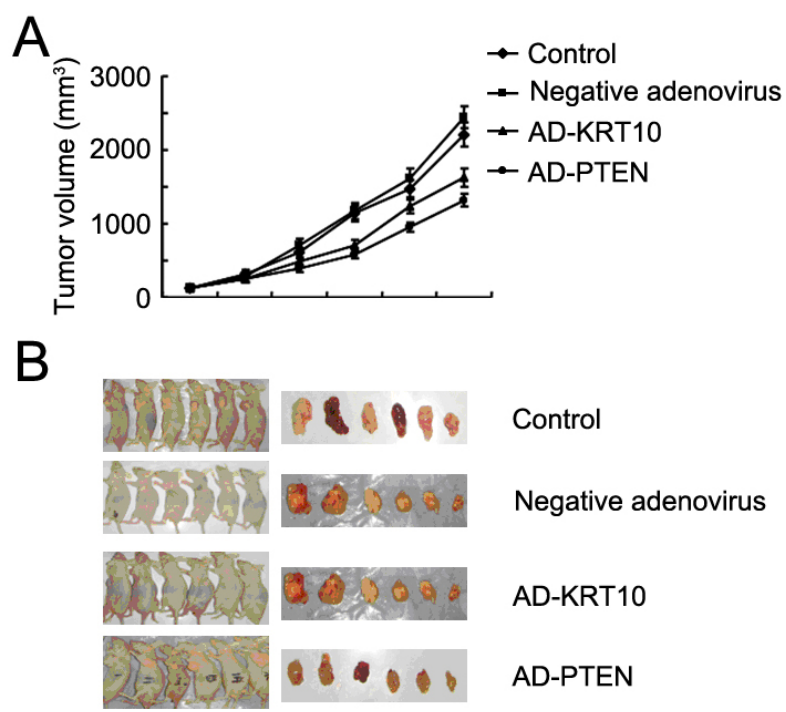

Figure 2. Recombinant adenovirus-mediated KRT10 and PTEN overexpression enhanced the in vivo antitumor efficacy of cisplatin. BALB/c nude mice bearing C13K xenograft tumors were injected with AD-KRT10, ADPTEN, and negative adenovirus and treated with cisplatin $24 \mathrm{~h}$ later. The growth curves of subcutaneous xenografts of $\mathrm{C} 13 \mathrm{~K}$ are shown (left panel). Representative tumors were removed and photographed (right panel).

\section{DISCUSSION}

Resistance to platinum-based chemotherapy is a major cause of treatment failure in human ovarian cancer. In previous studies, we showed that overexpression of PTEN can increase the sensitivity of human ovarian cancer cells to cisplatin. We also identified KRT10 as a downstream molecule of PTEN in C13K ovarian cancer cells after cisplatin treatment. In the current study, we showed that recombinant adenovirus-mediated PTEN or KRT10 overexpression improved the sensitivity of ovarian cancer cells to cisplatin both in vitro and in vivo, indicating that overexpression of PTEN and KRT10 may be a therapeutic method for the resistance of platinum-based chemotherapy in ovarian cancer.

PTEN and KRT10 have been reported to play important roles in cell cycle regulation, tumor cell apoptosis, and the regulation of cell adherence, migration, and differentiation (Cantley and Neel, 1999; Paramio et al., 1999; Reichelt et al., 2004). In addition, PTEN plays an important role in the reversal of MDR (Hou et al., 2010; Cassinelli et al., 2013). Overex- 
pression of PTEN can enhance the chemosensitivity of multiple cancer cells (Han et al., 2010; $\mathrm{Li}$ et al., 2013). In our previous study, overexpression of PTEN in C13K ovarian cancer cells improved the sensitivity to cisplatin, but not to paclitaxel. Furthermore, KRT10, which is present in numerous epithelial cells and tissues (Waters et al., 2009; Kobayashi et al., 2012), was identified as a downstream protein of PTEN in C13K/PTEN cells. In this study, we found that overexpression of PTEN or KRT10 mediated by recombinant adenovirus improved the antiovarian cancer effect of cisplatin in vitro and in vivo. These data suggest that PTEN and KRT10 are potential therapeutic targets for reversing the cisplatin resistance of ovarian cancer cells.

\section{Conflicts of interest}

The authors declare no conflict of interest.

\section{ACKNOWLEDGMENTS}

Research supported by the National Natural Science Foundation of China (\#30901742).

\section{REFERENCES}

Cantley LC and Neel BG (1999). New insights into tumor suppression: PTEN suppresses tumor formation by restraining the phosphoinositide 3-kinase/AKT pathway. Proc. Natl. Acad. Sci. U. S. A. 96: 4240-4245.

Cassinelli G, Zuco V, Gatti L, Lanzi C, et al. (2013). Targeting the Akt kinase to modulate survival, invasiveness and drug resistance of cancer cells. Curr. Med. Chem. 20: 1923-1945.

Dahia PL (2000). PTEN, a unique tumor suppressor gene. Endocr. Relat. Cancer 7: 115-129.

Han L, Zhang AL, Xu P, Yue X, et al. (2010). Combination gene therapy with PTEN and EGFR siRNA suppresses U251 malignant glioma cell growth in vitro and in vivo. Med. Oncol. 27: 843-852.

Hou R, Zhang J, Yin T, Cao H, et al. (2010). Upregulation of PTEN by peroxynitrite contributes to cytokine-induced apoptosis in pancreatic beta-cells. Apoptosis 15: 877-886.

Kobayashi T, Maruyama S, Abé T, Cheng J, et al. (2012). Keratin 10-positive orthokeratotic dysplasia: a new leucoplakiatype precancerous entity of the oral mucosa. Histopathology 61: 910-920.

Li D, Zhang Y, Xie Y, Xiang J, et al. (2013). Enhanced tumor suppression by adenoviral PTEN gene therapy combined with cisplatin chemotherapy in small-cell lung cancer. Cancer Gene Ther. 20: 251-259.

Li J, Yen C, Liaw D, Podsypanina K, et al. (1997). PTEN, a putative protein tyrosine phosphatase gene mutated in human brain, breast, and prostate cancer. Science 275: 1943-1947.

Li J, Zhang Y, Zhao J, Kong F, et al. (2011). Overexpression of miR-22 reverses paclitaxel-induced chemoresistance through activation of PTEN signaling in p53-mutated colon cancer cells. Mol. Cell Biochem. 357: 31-38.

Liu S, Yu M, He Y, Xiao L, et al. (2008). Melittin prevents liver cancer cell metastasis through inhibition of the Rac1dependent pathway. Hepatology 47: 1964-1973.

Paramio JM, Casanova ML, Segrelles C, Mittnacht S, et al. (1999). Modulation of cell proliferation by cytokeratins K10 and K16. Mol. Cell Biol. 19: 3086-3094.

Reichelt J, Furstenberger G and Magin TM (2004). Loss of keratin 10 leads to mitogen-activated protein kinase (MAPK) activation, increased keratinocyte turnover, and decreased tumor formation in mice. J. Invest. Dermatol. 123: 973-981.

Singh M, Chaudhry P, Fabi F and Asselin E (2013). Cisplatin-induced caspase activation mediates PTEN cleavage in ovarian cancer cells: a potential mechanism of chemoresistance. BMC Cancer 13: 233.

Smith BH, Gazda LS, Conn BL, Jain K, et al. (2011). Three-dimensional culture of mouse renal carcinoma cells in agarose macrobeads selects for a subpopulation of cells with cancer stem cell or cancer progenitor properties. Cancer Res. 71: 716-724.

Steelman LS, Navolanic PM, Sokolosky ML, Taylor JR, et al. (2008). Suppression of PTEN function increases breast cancer chemotherapeutic drug resistance while conferring sensitivity to mTOR inhibitors. Oncogene 27: 4086-4095.

Stordal B, Pavlakis N and Davey R (2007). A systematic review of platinum and taxane resistance from bench to clinic: an inverse relationship. Cancer Treat. Rev. 33: 688-703. 
Wan X, Li J, Xie X and Lu W (2007). PTEN augments doxorubicin-induced apoptosis in PTEN-null Ishikawa cells. Int. J. Gynecol. Cancer 17: 808-812.

Waters JM, Richardson GD and Jahoda CA (2009). Keratin 10 (K10) is expressed suprabasally throughout the limbus of embryonic and neonatal rat corneas, with interrupted expression in the adult limbus. Exp. Eye Res. 89: 435-438.

Wu H, Cao Y, Weng D, Xing H, et al. (2008). Effect of tumor suppressor gene PTEN on the resistance to cisplatin in human ovarian cancer cell lines and related mechanisms. Cancer Lett. 271: 260-271.

Yan X, Fraser M, Qiu Q and Tsang BK (2006). Over-expression of PTEN sensitizes human ovarian cancer cells to cisplatin-induced apoptosis in a p53-dependent manner. Gynecol. Oncol. 102: 348-355. 